\title{
A Modification to Homeopathic Drug Carcinosin
}

A crowdfunded biology project by Joshua Andrew

\section{June}

2018

\begin{abstract}
Objective:

This experiment was performed as an attempt to modify the homeopathic preparation Carcinosin in hopes to improve its effectiveness against cancer. The hypothesis is that the effectiveness of Carcinosin may be improved by creating the preparation from the same line of cells it will be used to treat. The experiment was performed in vitro (aka in cell cultures).
\end{abstract}

\section{Methods:}

Carcinosin 2.0 (name of experimental Carcinosin made in lab) was made from Colo 205 (human colorectal adenocarcinoma; a form colon cancer). First the a sample of $5 \mathrm{~mL}$ was taken from the culture of Colo 205 cells. Since Colo 205 cells grow both suspended and attached there are generally enough cells suspended in $5 \mathrm{~mL}$ of media to suffice. This sample was then centrifuged and suspended in $1 \mathrm{~mL}$ of new complete media. $200 \mu 1$ was then removed from the suspended cells and mixed with $200 \mu 1$ of Trypan blue. This mixture was then seeded into a hemocytometer and a viable cell count was obtained. After the amount of viable cells was determined, roughly 88,000 cells were suspended in $100 \mu 1$ sterilized DI water to produce a Mother Tincture (MT). All successions from MT onward, were done at a ratio of 1-part previous succession to 990 parts diluents. For example, MT to $1 \mathrm{BD}$ would be obtained by taking 1 part of BD and mixing it with 990 parts diluents. Each dilution was noted as BD (standing for Beneficium Dei). Up to 6BD was diluted using sterile DI water. After 6BD a mixture of $50 \%$ vegetable Glycerin: $50 \%$ DI water was used to derive the medicine 
to the appropriate potency. Once the appropriate BD was reached the medicine was kept at $4 \mathrm{C}$ until use and after use. Carcinosin 2.0 was both used on both Colo 205 and Hep G2 (human hepatocellular carcinoma; a form of liver cancer) to test for how the treatment would work on cells both of same line and different line. The results from Carcinosin 2.0 were tested against Carcinosin 200C. Viability was tested both through Trypan Blue Exclusion test and MTS assay. In the Trypan Blue Exclusion test, cells were exposed to drugs for 72 hours. In the MTS assay, cells were exposed to drugs for 24 hours.

\section{Results:}

Carcinosin 2.0 was observed to have significantly higher effects on decreasing cell viability than Carcinosin 200C on both COLO 205 and Hep G2 cell lines. When a neutral solution of the medium was made to the same dilution without cancer lines added it was seen to also have cytotoxic effects, though not as high as that of Carcinosin 2.0.

\section{Conclusion:}

This study opens up the possibility of the effectiveness of modifying Carcinosin. Further studies of the safety of the preparation and the effectiveness in animal models are warranted.

\section{Materials and Methods}

\section{Cell Lines}

The two cell lines used in this experiment were Hep-G2 (hepatocellular carcinoma: a common form of liver cancer) and Colo 205 (Duke's type D, colorectal; aka colon cancer). Hep-G2 was obtained from a graduate student during the culture's 7th passage. Colo 205 was obtained from the American Type Culture Collection (ATCC $\AA$ ). Both Hep-G2 and Colo 205 were cultured in Eagle's Minimum Essential Medium with 10\% Fetal Bovine Serum (FBS) and 1\% Penicillin-Streptomycin. Eagle's Minimum Essential Medium and FBS were 
obtained from the ATCC $®$. Penicillin-Streptomy was obtained from VWR Corporation ${ }^{\circledR}$. Cells were maintained at $37 \mathrm{C}$ in a $5 \% \mathrm{CO}_{2}$ incubator.

\section{Homeopathic Remedies}

Carcinosin 200C was obtained from Helios Homeopathy. Vegetable Glycerin was obtained from Bulk Apothecary. Sterile deionized (DI) water and Glycerin were autoclaved in order to sterilize them.

The Carcinosin 2.0 was produced by the following method:

1. Cancer cells from Colo 205 were collected via a $5 \mathrm{~mL}$ sample of media. Colo 205 grows both attached and suspended, therefore a $5 \mathrm{~mL}$ sample has enough viable cells for the experiment.

2. The $5 \mathrm{~mL}$ sample was centrifuge and old media was removed. The cells were then suspended in $1 \mathrm{~mL}$ of complete media. $200 \mu 1$ of the suspended cells were then stained with $200 \mu 1$ of Trypan Blue.

3. This mixture of cells and Trypan Blue were seeded into a hemocytometer and a viable cell count was obtained. Roughly 88,0000 live cells were counted and diluted in $100 \mu 1$ of sterilized DI water to produce a Mother Tincture.

4. The Mother Tincture (MT) (mixture of $100 \mu 1$ of sterilized DI water and 88,000 Colo 205 cells) was either pipetted up and down 10 times or spun using a vortex at speed of 5 for 10 seconds. These two methods appeared to work equally well. Both methods were used in place of the classic method of shaking the vial used by Samuel Hahnemann (founder of Homeopathy) to give a more accurate way to quantify the force used to mix each succession of the drug.

5. Due to a clerical error, successions were done at a 1-part previous succession to 990 parts diluents. This rate of succession was seen to be effective and used for the experiment. The dilution is denoted as BD (standing for Beneficium Dei). MT was diluted using DI water to the potency equivalent to that of 1BD. 
Up to $6 \mathrm{BD}$ was diluted using sterile DI water. After 6BD a mixture of $50 \%$ vegetable Glycerin: 50\% DI water was used to derive the medicine to the appropriate potency. The reason water is used up to 6BD is that in "Scientific Method of Preparing Homoeopathic Nosodes," (see lab note titled "Medical Journals: Carcinosin") water is a preferred vehicle up to the $6^{\text {th }}$ dilution. Also, is specified in the same journal that alcohol "denaturation of proteins, distortion from the original form, and probably loss of antigenicity" when exposed to heat such as incubation. 990 parts of the previous BD was removed, leaving 1 part behind. 990 parts of either DI water or DI water/Vegetable Glycerin mix was added to the 1 part and either spun or pipetted. After the MT was made the Carcinosin 2.0 was switched into a new vial for both MTS tests and Trypan Blue tests. In the Trypan Blue Exclusion test, the drug switched vials at the potencies of $6 \mathrm{BD}, 32 \mathrm{BD}$, 100BD, and 121BD. In the MTS test, the drug changed vials at the potencies of 6BD and 32BD. These switches in vials were to save the succession that was made before the BD of the drug was reached. Outside of the potencies given above, Carcinosin 2.0 was made in the same vial.

1. Carcinosin 2.0 was stored in a refrigerator at $4{ }^{\circ} \mathrm{C}$ and was stored for 2 months with no changes in effectiveness observed.

A blank drug was made using the above method, except for the addition of the cancer cells. In other words, it consisted of only DI water and Vegetable Glycerin. Carcinosin 2.0 in this experiment was derived from Colo 205 cells.

\section{Viability Tests}

\section{MTS Assay}

The MTS Assay was obtained from Promega (product name on company site CellTiter 96® AQueous One Solution Cell Proliferation Assay). Whenever the cells were not being directly worked with, they were in the incubator at $37^{\circ} \mathrm{C}$ and $5 \% \mathrm{CO}_{2}$. MTS assay is a viability assay (or test) that determines the number of viable cells by color change in the media after it is added.

The MTS assay followed the following procedures: 
1. Cells were stained with Trypan Blue solution and counted by hand using a hemocytometer. After accessing the viability, an appropriate number of cells were extracted from the main cultures. These allocates were then spun down in a centrifuge and resuspended to appropriated density in Eagle's Minimum Essential Medium with 10\% FBS and 1\% PenicillinStreptomycin.

a. For Colo 205 sample, the density was 820 cells per $100 \mu \mathrm{L}$.

b. For Hep G2 sample, the density was 6200 cells per $100 \mu \mathrm{L}$.

2. The resuspended allocates were used to seed a 96 well plate. $100 \mu \mathrm{L}$ of the allocate was used per well. Hep G2 and Colo 205 were placed in separate 96 well plates to protect from contamination.

3. After seeding, the cells were allowed to rest for 24 hours. This helped ensure there was no contamination present.

4. After 24 hours, Carcinosin 200, Carcinosin 2.0, and the blank drug were distributed among the cells. The quantities given were $5 \mu \mathrm{L}$ and $24 \mu \mathrm{L}$ per well for each of the above. One set of wells was allowed to continue to grow, without any drugs added, to function as a positive control.

5. Cells were allowed to rest for 24 hours with drug present in the media.

6. After 24 hours, old media was pipetted off of all wells (including the positive control). $100 \mu \mathrm{L}$ of new media, consisting of Eagle's Minimum Essential Medium with 10\% FBS and 1\% Penicillin-Streptomy, was added. A set of wells were seeded with the $100 \mu \mathrm{L}$ of the same media to function as blank wells.

a. Blank wells are used to determine background absorbance that occurs when a cell plate is read by a cell plate reader. This background absorbance is subtracted from the absorbance of all other wells.

7. Cells were allowed to rest in the new media for 24 hours.

8. After 24 hours, $10 \mu \mathrm{L}$ of MTS Assay was added to each well. After MTS was added, cells were incubated until significant color change was observed (1-2 hours, for this experiment). Once significance color change was observed, plates were read, using a cell plate reader set to 490 wavelengths.

9. Once plate reader was done and absorbance values were obtained, cell plates were disposed. 
See Lab note titled "Trypan Blue Exclusion Assay" for the procedure used for the Trypan Blue Exclusion Assay.

\section{Results}

\section{MTS Assay}

Cell viability after treatment

\begin{tabular}{|l|c|c|c|c|c|c|}
\hline & \multicolumn{2}{|c|}{ Carcinosin 200C } & \multicolumn{2}{c|}{ Carcinosin 2.0 } & \multicolumn{2}{c|}{ Blank Drug } \\
\hline Cell Line & $5 \mu \mathrm{L}$ & $24 \mu \mathrm{L}$ & $5 \mu \mathrm{L}$ & $24 \mu \mathrm{L}$ & $5 \mu \mathrm{L}$ & $24 \mu \mathrm{L}$ \\
\hline Colo 205 & $93 \%$ & $75 \%$ & $27.3 \%$ & $3.7 \%$ & $29.2 \%$ & $8.9 \%$ \\
\hline HepG2 & $120 \%$ & $20 \%$ & $81.1 \%$ & $5.7 \%$ & $65.8 \%$ & $17.6 \%$ \\
\hline
\end{tabular}

The percentages listed above are the number of viable cells left after the MTS assay procedure was followed. For example, Carcinosin 2.0 at $24 \mathrm{uL}$ in Colo 205 cells lead to $3.7 \%$ viable cells left.

\section{Trypan Blue Exclusion Test}

See Lab note titled "Trypan Blue Exclusion Assay" for the result of the Trypan Blue Exclusion Assay. This assay was used as a preliminary test of the drug's effectiveness at three different BD potencies.

\section{Conclusion and Discussion}

In this experiment, it was observed that Carcinosin can be altered in such a way that it becomes more effective. Carcinosin 2.0 was seen to perform better on the strand of cells that it was derived from when the amount of drug given was low. At high amounts, Carcinosin 2.0 was observed to work relatively equally on the cell line it was derived from (Colo 205) as well as the cell line it was not derived from (Hep G2).

This means that when distributed in lower concentrations, the drug is more specific to the cell line it is derived from which in this case is Colo 205. Also, this means that when distributed in higher amounts the drug appears to not be as specific in provoking apoptosis (cellular death). 
In this experiment, the blank drug seemed to closely mimic Carcinosin 2.0. Journals that had previously used Carcinosin (see lab note titled "Medical Journals: Carcinosin" for journals), and created a blank drug (also known as a vehicle) were re-evaluated. In those journals, it was observed that the blank drug used was not diluted in successions at all, but was rather a set amount of the solvents (alcohol and water) mixed together. This could possibly mean that the blank drug in this experiment was so effective because it was created in the same manner as the drug in question.

Going forward, it is my hope that others will seek to replicate this experiment. It is also my hope that others will expand upon its findings in order to either verify or disprove, the findings in accordance with their own data. 